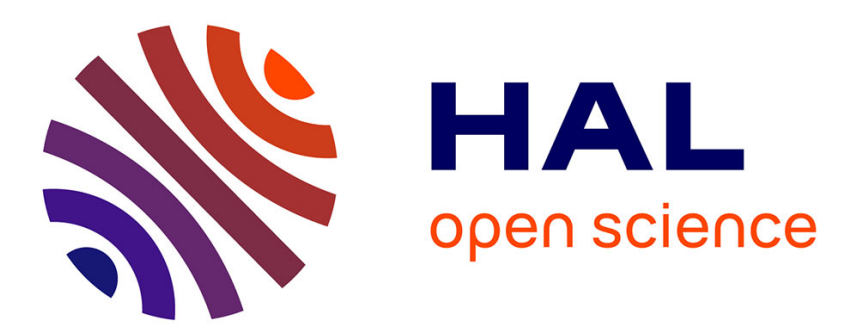

\title{
A distribution network design problem in the automotive industry: MIP formulation and heuristics
}

Mouna Kchaou Boujelben, Céline Gicquel, Michel Minoux

\section{To cite this version:}

Mouna Kchaou Boujelben, Céline Gicquel, Michel Minoux. A distribution network design problem in the automotive industry: MIP formulation and heuristics. Computers and Operations Research, 2014, 52, pp.16-28. 10.1016/j.cor.2014.07.007 . hal-01120249

\section{HAL Id: hal-01120249 \\ https://hal.science/hal-01120249}

Submitted on 29 Mar 2016

HAL is a multi-disciplinary open access archive for the deposit and dissemination of scientific research documents, whether they are published or not. The documents may come from teaching and research institutions in France or abroad, or from public or private research centers.
L'archive ouverte pluridisciplinaire HAL, est destinée au dépôt et à la diffusion de documents scientifiques de niveau recherche, publiés ou non, émanant des établissements d'enseignement et de recherche français ou étrangers, des laboratoires publics ou privés. 


\title{
A distribution network design problem in the automotive industry: MIP formulation and heuristics
}

\author{
Mouna Kchaou-Beljeben ${ }^{1}$, Céline Gicquel ${ }^{2}$, Michel Minoux ${ }^{3}$ \\ ${ }^{1}$ Laboratoire Génie Industriel, Ecole Centrale Paris, Paris, France \\ ${ }^{2}$ Laboratoire de Recherche en Informatique, Université Paris Sud, Orsay, France \\ ${ }^{3}$ Laboratoire d'Informatique de Paris 6, Université Pierre et Marie Curie, Paris, France
}

Working paper submitted for publication in Computers \& Operations Research May 2014 


\title{
A distribution network design problem in the automotive industry: MIP formulation and heuristics
}

\begin{abstract}
We consider a multi-product distribution network design problem arising from a case-study in the automotive industry. Based on realistic assumptions, we introduce minimum volume, maximum covering distance and single sourcing constraints, making the problem difficult to solve for large-size instances. We thus develop several heuristic procedures using various relaxations of the original MIP formulation of the problem. In our numerical experiments, we analyze the structure of the obtained network as well as the impact of varying the problem parameters on computation times. We also show that the implemented heuristic methods provide good quality solutions within short computation times on instances for which a state-of-the-art MIP solver does not produce any feasible solution. Keywords: Supply chain network design, Location-routing, Minimum volume constraints, Linear relaxation
\end{abstract}

\section{Introduction}

A considerable part of a company costs is devoted to the supply chain management, in particular logistics operations such as transport and storage. For instance, according to the CSCMP (Council Of Supply Chain Management Professionals) annual reports [9], the logistics costs as a percentage of the GDP in the USA varied between $7.9 \%$ and $9.9 \%$ in the last decade. This is why more attention and consideration has to be given to optimizing the supply chain planning. One key question at the strategic planning level is designing the supply chain network and more precisely locating the major facilities of the company, namely plants and distribution centres. In the present work, we deal with a multi-product distribution network design problem arising from a case-study in the automotive industry. The planning horizon that we consider is not very long (one year in our case-study). The overall network structure consists of three levels: assembly plants in the first level, distribution centres (DCs) in the second one and customers (car dealers) in the third one. We assume that the number and location of the plants as well as the number and location of the customers are fixed. Given a deterministic demand of customers for each product and a list of potential DCs, our main concern is to locate DCs and to assign customers to them in such a way as to minimize the total distribution costs. In order to evaluate transport routes from DCs to customers, we construct groups of customers (clusters) using a clustering-based location-routing approach which allows dealing with large-size real-life instances. Moreover, we consider realistic hypotheses that have not been simultaneously taken into account in the literature. Namely, we introduce single sourcing restrictions and minimum volume constraints for both transport flows and facility throughputs. We also use maximum covering distance constraints, i.e. the length of the route between a DC and any cluster that it serves (computed as the length of the shortest route starting from the DC, visiting all the customers of the cluster then coming back to the DC) must not exceed a given distance. The combination of these operational features involves seeking a trade-off between conflicting objectives, thus the problem becomes difficult to solve for large-size instances (for some instances it is even 
difficult to find feasible solutions). This is why we develop efficient heuristic methods based on various relaxations of the original mixed integer programming (MIP) formulation of the problem. To the best of our knowledge, it is the first time that this kind of approach is applied to determine both location and assignment variables in a supply chain network design problem subject to minimum volume and distance constraints.

The paper is organized as follows. In the next section, we propose an overview of the related literature. Section 3 is devoted to the explanation of the modeling considerations and to the description of the mathematical formulation of the problem. Then, efficient heuristic procedures are investigated in section 4 and computational results concerning our case-study are presented in section 5. Finally, some conclusions are provided in section 6 .

\section{Position in the literature}

Our research is related to two main literature streams, the first one being facility location and the second one supply chain network design. This is why we first propose an overview on these expanding fields. We then discuss the integration of minimum volume and distance constraints in supply chain network design problems.

\subsection{Facility location and supply chain network design}

In a classical facility location problem, the objective is to locate new facilities and to determine the related product flows on a given network, where the locations of demand points are known. In the present work, we focus on a discrete location problem, i.e. facility locations have to be chosen among a list of eligible sites. A survey on discrete location science can be found, among other, in [25].

A typical approach in supply chain network design problems is the use of integrated models, i.e. simultaneously modeling and solving the strategic location problem and other operational issues. This body of works includes production-distribution problems [32], location-inventory problems [3] and location-routing ones (LRP) [24]. In the context of our study, we were particularly interested in the latter problem; see e.g. [22] for an overview on the classification of LRP. From the related literature, we can mainly identify three different approaches when dealing with vehicle routing within supply chain network design problems. The first one can be called the explicit method as routes are explicitly modeled as decisions in the optimization problem (see e.g. [33]) but this may result in the formulation of large-size MIP, most of which are hard to solve. The second method consists in using the continuous approximation, i.e. expressing continuous functions to characterize the customer demand distribution and their locations (for instance in [29] continuous approximation is used to approximate the optimal routing costs). The major disadvantage in this case is the need to resort to strong assumptions such as a uniform demand distribution or a uniform customer density. Finally, it is also possible to use a sequential clustering-based method, as proposed by Barreto et al. in [5]. We chose to apply this method in order to have a good approximation of the routing costs while keeping a manageable size for the optimization problem. This allows dealing with real-life instances involving many customers.

\subsection{Integration of minimum volume constraints and maximum distance constraints}

One of the constraints involved in our model is the maximum covering distance constraint. A customer is said "covered" if there exists an opened facility situated within a pre-specified distance of it. In order to formulate this 
kind of distance constraints, we can either use covering objectives (mainly when locating public sites such as schools, police stations, hospitals, parks, etc.) or impose covering distance constraints with any type of objective, usually cost or distance minimization. The literature dealing with covering objectives is abundant (see [10] for a recent literature review) whereas only a few papers, among which are [20], [27], [1] and [21], consider covering distance constraints.

In our network design model, a major feature is imposing a minimum volume on each transport link and on each opened DC. This is an approach used in facility location and supply chain network design to introduce flow consolidation. The literature devoted to this stream appears to be relatively scarce. Table. 1 provides a summary of the main characteristics of the seventeen papers that we found in this field. From a modeling point of view, we can first notice that only four works consider minimum volume constraints for transport quantity on each link of the network. Most of the papers deal with this kind of constraint for the throughput of facilities and none of them propose a model using minimum volume constraints for both facility throughput and transport quantities. Multiproduct or single sourcing are restrictions that could make the problem more difficult to solve as the former increases the variable number and the latter adds 0-1 constraints. Only two of the listed works ([11] and [26]) simultaneously consider the multi-product and single sourcing features. From a solution method perspective, the computational results provided in [26] concern only small instances (27 binary variables) and use a commercial solver. Other papers implement heuristic solution procedures, mainly constant approximation [12, 13, 15, 19, 30] and linear relaxation based heuristics $[6,16,18,31]$. In the latter papers, the heuristic strategy is based on rounding fractional solutions to 0 or 1 , depending on their values. In [16], the idea consists in rounding to 1 the carrier selection variable having the greatest fractional value. In the other works, the rounding of a location variable depends on the comparison of its fractional value to an upper bound close to 1 and a lower bound close to 0 . None of the four papers focuses on rounding assignment variables either because they are not problematic in the solution procedure or because they are continuous variables.

The present work features four main contributions as compared to the above-mentioned works. First, to fit reallife requirements, we consider maximum distance and single sourcing restrictions simultaneously with minimum volume constraints when modeling the assignment variables for multiple products. Second, in our study, we assign minimum volume constraints to facility throughputs as well as to transport flows, which makes the problem more realistic but increases the difficulty of solving it. Third, we propose MIP-based heuristic procedures using various types of linear relaxations to determine location and assignment variables (both sets of variables being required to be binary). Finally, we carry out numerical experiments using a case-study with large-size instances (16 assembly plants, 51 potential DCs and 448 car dealers).

\section{Problem modeling}

\subsection{Problem description}

We study a three-level distribution network consisting of assembly plants, distribution centres (DCs) and customers (see. Fig. 1). Cars are produced at assembly plants and are then transported by truck to intermediate distribution centres (via "primary" transport links) before being sent by truck to the car dealers (via "secondary" 


\begin{tabular}{|c|c|c|c|c|c|c|c|c|c|c|c|}
\hline \multirow[t]{2}{*}{ Paper } & \multirow[b]{2}{*}{ 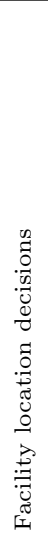 } & \multirow[b]{2}{*}{ 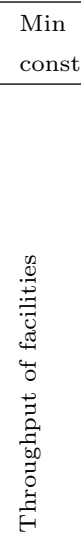 } & \multirow[b]{2}{*}{ 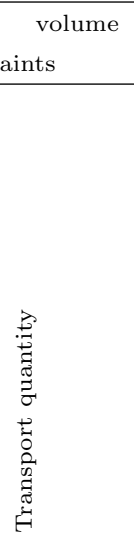 } & \multicolumn{2}{|c|}{$\begin{array}{l}\text { Complicating } \\
\text { features }\end{array}$} & \multicolumn{6}{|c|}{$\begin{array}{l}\text { Solution procedure and numerical } \\
\text { results }\end{array}$} \\
\hline & & & & 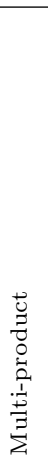 & 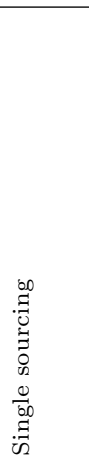 & 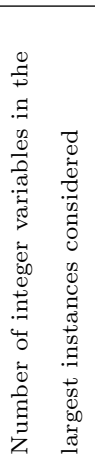 & 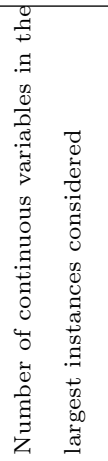 & 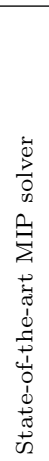 & 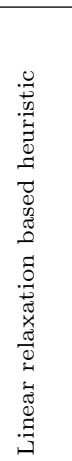 & 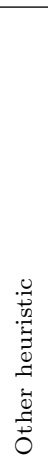 & 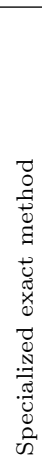 \\
\hline Krumke and Thielen (2011) [15] & & & $\mathrm{X}$ & & & $\mathrm{NM}$ & NM & & & $\mathrm{X}$ & $\mathrm{x}$ \\
\hline Lim et al. (2006) [16] & & & $\mathrm{X}$ & & & 10890 & 0 & & $\mathrm{x}$ & $\mathrm{X}$ & $\mathrm{x}$ \\
\hline Seedig (2011) [28] & & & $\mathrm{x}$ & & & 50 & 50 & & & & $\mathrm{x}$ \\
\hline Zhu et al. (2011) [34] & & & $\mathrm{X}$ & & & 119643 & 119643 & $\mathrm{x}$ & & & \\
\hline Alumur et al. (2012) [2] & $\mathrm{X}$ & $\mathrm{X}$ & & $\mathrm{X}$ & & 1200 & 58000 & $\mathrm{x}$ & & & \\
\hline Barros et al. (1998) [6] & $\mathrm{x}$ & $\mathrm{X}$ & & $\mathrm{x}$ & & NM & NM & & $\mathrm{X}$ & & \\
\hline Correia et al. (2013) $[8]$ & $\mathrm{x}$ & $\mathrm{x}$ & & $\mathrm{x}$ & & 1728 & 83705 & $\mathrm{x}$ & & & $\mathrm{x}$ \\
\hline Geoffrion and Graves (1974) [11] & $\mathrm{x}$ & $\mathrm{x}$ & & $\mathrm{x}$ & $\mathrm{x}$ & 727 & 23513 & & & & $\mathrm{x}$ \\
\hline Guha et al. (2000) [12] & $\mathrm{x}$ & $\mathrm{x}$ & & & $\mathrm{x}$ & NM & $\mathrm{NM}$ & & & $\mathrm{x}$ & \\
\hline Karger and Minkoff (2000) [13] & $\mathrm{x}$ & $\mathrm{x}$ & & & $\mathrm{x}$ & NM & $\mathrm{NM}$ & & & $\mathrm{x}$ & \\
\hline Melo et al. (2005) $[17]$ & $\mathrm{x}$ & $\mathrm{X}$ & & $\mathrm{x}$ & & 270 & 732810 & $\mathrm{x}$ & & & \\
\hline Melo et al. (2011) [18] & $\mathrm{x}$ & $\mathrm{x}$ & & $\mathrm{x}$ & & 560 & 107057 & $\mathrm{x}$ & $\mathrm{X}$ & & \\
\hline Meyerson (2001) [19] & $\mathrm{x}$ & $\mathrm{x}$ & & & $\mathrm{X}$ & NM & NM & & & $\mathrm{x}$ & \\
\hline Ndiaye and Alfares (2008) [23] & $\mathrm{x}$ & $\mathrm{x}$ & & & $\mathrm{X}$ & 370 & 1810 & $\mathrm{x}$ & & & \\
\hline Sabri and Beamon (2000) [26] & $\mathrm{x}$ & $\mathrm{x}$ & & $\mathrm{x}$ & $\mathrm{x}$ & 27 & 214 & $\mathrm{x}$ & & & \\
\hline Svitkina $(2010)[30]$ & $\mathrm{x}$ & $\mathrm{x}$ & & & $\mathrm{x}$ & NM & NM & & & $\mathrm{X}$ & \\
\hline Thanh et al. (2010) [31] & $\mathrm{x}$ & $\mathrm{x}$ & & $\mathrm{X}$ & & 2185 & 30870 & $\mathrm{x}$ & $\mathrm{x}$ & & \\
\hline Present work & $\mathrm{X}$ & $\mathrm{X}$ & $\mathbf{X}$ & $\mathbf{x}$ & $\mathbf{X}$ & 62832 & 1632 & $\mathbf{x}$ & $\mathbf{x}$ & & \\
\hline
\end{tabular}

Table 1: Literature review on facility location and network problems featuring minimum volume constraints (NM=Not Mentioned).

or "last-mile" transport links). Products to be distributed may wait on manufacturing sites or on DCs until they are shipped but a maximum waiting time at each facility is imposed in order to reduce the total delivery time. In the present work, this maximum waiting time is considered as given for each plant and each DC; it typically amounts to a few days.

The company chose an organization using DCs to benefit from economies of scale for long-distance transport from manufacturing sites to DCs. In fact, starting from a given plant, shipments to various customers are consolidated and sent to a given DC that serves these customers. This allows achieving full truckload transport from plants to DCs and leads to meeting the targeted shipping frequencies. On the contrary, if direct transport from plants to customers were used, serving "small" customers would be problematic: either less-than-truckload transport would be used or waiting times would increase, which means raising transport costs or deteriorating customer service.

Around 35 distinct car types, without considering colors and options, have to be distributed through the network. A given plant can manufacture different car types and a given car type can be manufactured in several sites. Nevertheless, we assume that the demand of each car dealer for the different car types have already been assigned to assembly plants. This is indeed a strategic decision made by high management and not at the distribution level. Demands are thus expressed as quantities to be distributed from a given source (assembly plant) to a given 


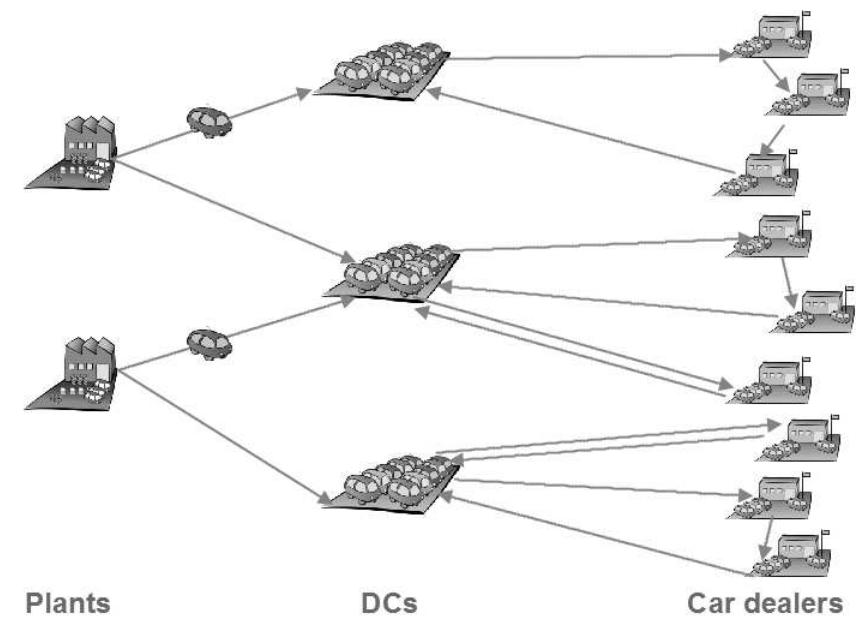

Figure 1: Car distribution network

destination (car dealer).

Distribution costs involve the costs of primary transport, secondary transport and the transit through DCs. These costs are obtained by multiplying unit costs by quantities. No inventory costs are included as only maketo-order products are considered in the study. Moreover, as the management of the distribution centres and the related activities are outsourced to logistics suppliers, there are no fixed opening costs to be charged to the car maker. There is only a unit transit cost (considered as given in our study) to be paid to the logistics supplier each time a car goes through a DC. The unit transport cost on each transport link is computed by dividing the cost of a truck by its load. The cost of a truck depends on the traveled distance, which consists for primary transport in the length of a direct trip from a plant to a DC. For secondary transport, it is equal to the length of the shortest route starting from a DC, visiting a cluster of customers then coming back to the DC.

To make the model as close to reality as possible, these are the operational constraints that we also consider:

- Maximum covering distance constraints: as mentioned in $\S 1$, a DC cannot be situated farther than a given distance from a cluster that it serves. In fact, deliveries are usually made by drivers that have to come back to the distribution centre at the end of the working day. The traveled distance per delivery trip thus should not be greater than a given limit, allowing the driver to comply with the legal daily driving time. In the reference dataset used in $\S 5.1$, the value of the maximum covering distance parameter is computed as the driving time multiplied by a constant average speed.

- Minimum volume constraints on transport links: maximizing the loading of trucks is one of the priorities in car distribution as cars are voluminous products transported by specific trucks with limited capacities, typically, a truck can carry up to 8-10 small cars. Thus, if it is not fully loaded, the unit cost per transported car could significantly increase. Products to be distributed to the same destination have to wait on the plant or the distribution centre until a full truckload is consolidated. If the total flow rate on a given transport link is low, the waiting time could be considerable and lead to excessive lead times. In this case, the best solution is to consolidate many flows on a same transport link. This is expressed in our model using minimum volume constraints that condition the use of primary and secondary transport links. The lower bound assigned to 
each link has to be greater than the volume ensuring on average a full truckload within a fixed maximum waiting time allowed at the sourcing point. Using this lower bound, we can assume that the transport of products is made through full truckloads and thus the unit transport cost on the link is computed as the cost of a truck divided by the truck capacity. Exact definitions of the parameters involved in the minimum volume constraints can be found in $\S 3.4 .1$.

- Minimum volume and maximum capacity constraints on DCs: The unit transit cost above mentioned results from a commercial negotiation between the car maker and the logistics supplier managing the DC. It only applies if the total throughput of the DC is between a minimum volume and a maximum capacity.

- Single sourcing restrictions: for a given cluster, all the products manufactured in a same plant should go through a single DC. This is an operational feature that facilitates day-to-day operations. Thus, it is meaningful to use an aggregate representation of the product types based on their sourcing plants. In the sequel, we consider the different types produced in a same plant as a single product, which reduces the number of products from 35 to 16 . The model keeps however its multi-product feature as assignments of clusters to DCs can differ according to the sourcing plant.

Aggregation of products per plant and the use of mixed product loads on primary (plant-DC) transport links lead to considering average capacities for trucks from plants to DCs. We propose to use an average truck capacity $\left(W_{i}\right)$ specific to each plant $(i)$ and to compute it as the weighted average load factor over the whole demand of the various product types manufactured in the given plant with the demand of each product type as weight. Notice that the load factor of a car type is the number of cars of this type that can be loaded on a truck. It can differ according to the car size.

On secondary transport links, trucks of mixed product types are also shipped from DCs to clusters of customers. As we do not know in advance what kind of product will be transported, we propose to use a common weighted average capacity $W$ over all the car types.

The simultaneous introduction of minimum volume, maximum covering distance and single sourcing constraints makes the problem closer to real-life requirements but leads to several computational difficulties. Before providing the detailed mathematical formulation of the network design problem and presenting a heuristic solution method to solve it, we first explain the proposed clustering-based location-routing approach. Then, we analyze the main trade-offs to be achieved regarding the distribution network structure.

\subsection{The clustering-based location-routing approach}

By introducing a minimum volume constraint for each transport link, we implicitly assume that the total demand of the destination point (DC or customer) exceeds the transport minimum volume. For primary transport, this is not problematic as a given DC is supposed to deal with the demand of many customers for many product types. For secondary transport, this is not obvious. The demand of some car dealers could be below the threshold corresponding to reaching a full truckload within the maximum waiting time allowed at a DC. This is why the company resorts to grouping deliveries: a given truck starting from a distribution centre may have to visit several customers before coming back to the distribution centre. We have then to answer the following question: which customers to serve 
together in the same route? This leads to a Vehicle Routing Problem (VRP). In the present work, we propose to use a clustering-based location-routing approach to obtain a good approximation of secondary transport routes without increasing the problem size. In fact, using an integrated location-routing approach (LRP) would be expensive in terms of computational effort, in particular for real-life instances. To the best of our knowledge, no instances of uncapacitated LRP that are significantly larger than those in [4] (with 199 customers and 14 DCs) have been exactly solved in the literature, whereas for capacitated LRP, the largest instances involve only 100 customers and 10 DCs (see the same work). Given that we deal in our case-study with about 500 customers, 15 products and 50 capacitated DCs, considering an exact location-routing approach would be computationally intractable. This is why we develop a sequential procedure.

\subsubsection{Main idea}

The idea of the clustering-based method is to form groups of customers, which will be allocated to the same truck routes. Fig. 2 illustrates the clustering-based method that we can summarize in four steps:

1. Construct clusters of customers lying close to one another, while complying with a minimum volume limit and some other constraints.

2. Solve a Traveling Salesman Problem (TSP) for each distribution route from DCs to clusters.

3. Compute the unit cost per transported product for each route.

4. Solve a distribution network design problem using clusters as customers instead of the original car dealers.

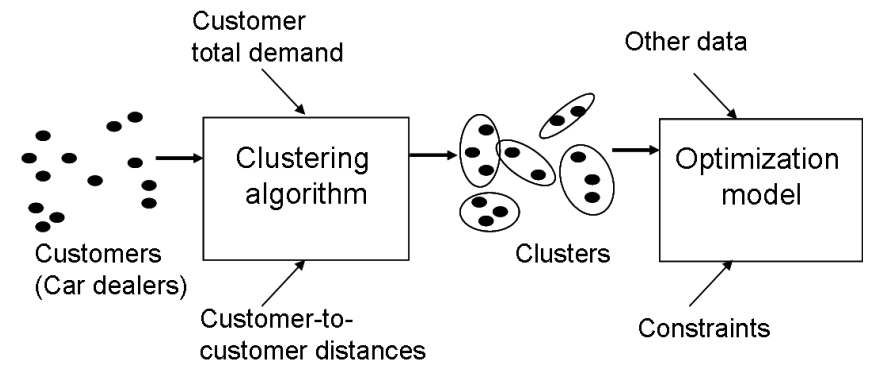

Figure 2: The clustering based approach

In step 1, we consider as input of the clustering algorithm the total demand of each customer, including all product types. This is due to the possible combination of different car types in a same secondary transport truckload. In step 2, we use a simple enumeration procedure to determine the shortest route starting from a DC, visiting all the customers of a cluster then coming back to the DC. In fact, a particular feature of car distribution is that a route consists of a small number of customers (usually between one and three). This makes solving the TSP problem through complete enumeration possible. In step 3, we use a classical formula with fixed and kilometric components to compute the cost of a truck for a given route. Then, we divide the truck cost by the average truck capacity to deduce the unit transport cost.

When using this sequential approach, we have to ensure consistency between the clustering step and the optimization step. We have then to make sure that the minimum volume required for each cluster is greater than the minimum volume required for secondary transport. 


\subsubsection{Algorithm}

The proposed algorithm is a heuristic approach which consists in grouping the close customers into clusters (an assessment of the quality of the solutions produced by the heuristic clustering is provided in $\S 5.3 .2)$.

The maximum number of customers per cluster is imposed by the decision maker and has to be strictly met by the algorithm. The total demand of a cluster has to be greater than a given lower bound. For instance, we can consider as a lower bound, the volume ensuring on average one or two full truckloads every week (if the maximum waiting time allowed at DCs is one week). The clustering produced by the algorithm has also to meet, as far as possible, two other constraints: a maximum total demand per cluster and a maximum distance between any two customers of a cluster. The first constraint is added in the first phase of the clustering to balance the total demand over the clusters. This could prevent for instance from grouping two customers already having a demand greater than the minimum required quantity. The second constraint is used in the first phase of the clustering to impose a proximity between the customers of a same cluster. However, these two constraints are not mandatory and may be relaxed in order to find feasible solutions.

Input distances between customers are calculated using a Geographic Information System (GIS) to form a point-to-point distance matrix. Algorithm 1 illustrates the pseudo-code of the first phase of the implemented algorithm. At the beginning of the procedure, the cluster list is initialized to single-element clusters (i.e. each customer corresponds to a cluster). We chose the following distance definition between two clusters p and q: $\operatorname{Distance}(p, q)=\operatorname{Min}\left\{\operatorname{Distance}(i, j), i \in C_{p}, j \in C_{q}\right\} . C_{p}$ is the set of customers of the cluster p. This proximity measure is called the "single linkage" measure; see [5] for a discussion of other possible measures.

Once the first phase of the clustering algorithm has been completed, a second phase has to be applied in order to consider the clusters discarded in the first phase due to the different constraints. In the second phase, only the minimum volume and the maximum number of customers constraints are imposed. We check that each cluster has a total demand greater than the minimum demand required. If it is not the case for some cluster $q$ then we attach each of its customers to the nearest cluster having fewer customers than the maximum allowed number of customers.

\subsection{Main trade-off analysis}

Before detailing the mathematical formulation of the optimization problem under study, we first analyze the main trade-offs to be achieved. These trade-offs which are mainly driven by minimum volume and maximum distance constraints not only influence the number of opened DCs but also the existence of a feasible solution.

In classical facility location problems, one of the main purposes of optimization is to decide about the number of DCs to open so as to achieve the best possible trade-off between fixed-charge opening costs and transport costs. In our problem, in addition to minimizing transport costs, we have also to cope with maximum covering distance constraints. This could lead to opening many DCs in order to be close to customers. As previously mentioned, no fixed-charge opening costs are included in the proposed model but minimum quantities are required. Thus, opening a DC at each potential location may not be feasible. It would result in splitting the global volume to be distributed from the plants in many DCs so that both the constraints of minimum throughputs and the constraints of minimum volume on primary transport links are likely to be violated in the corresponding solution. One of the purposes of the 
proposed optimization model is thus to build a network so as to meet the maximum covering distance constraints and to minimize the total transport costs while ensuring that minimum volume constraints are satisfied.

Furthermore, experiences with typical parameters and data of our case-study show that it is not always possible to comply with minimum volume constraints for primary transport links in the presence of maximum covering distance constraints. This means that shipping fully loaded trucks from plants to distribution centres within tight waiting times is not always feasible. Therefore, in the model, we have considered the possibility of violating the strict minimum volume constraints on primary transport links but keeping total constraint violation as low as possible via penalties (see $\S 5.3 .1$ for details about the choice of the penalty coefficients).

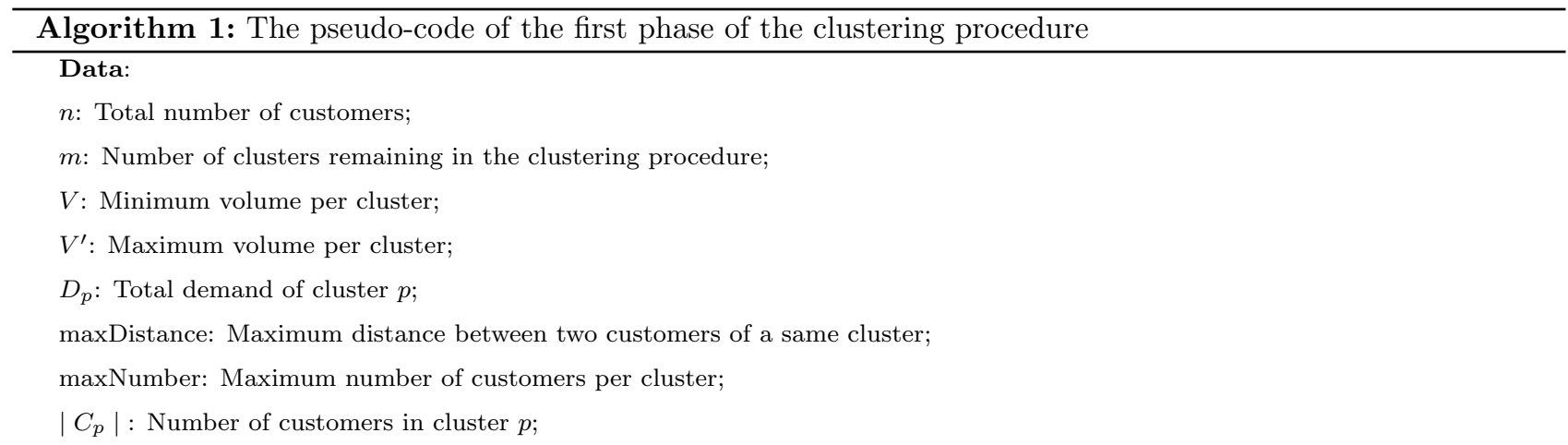

\section{Algorithm:}

Initialize the $n$ customers to $n$ individual clusters;

Initialize $\mathrm{m}=\mathrm{n}$;

while $m>1$ do

Find the pair of authorized clusters $(p, q)$ such as distance $(p, q)$ is the minimum of the distance matrix;

if $\operatorname{distance}(p, q) \leq \operatorname{maxDistance}$ then

if $\left|C_{p}\right|+\left|C_{q}\right| \leq$ maxNumber then

if $D_{p}+D_{q} \leq V^{\prime}$ then

Merge clusters $p$ and $q$;

$\mathrm{m}=\mathrm{m}-1$;

if $D_{p}+D_{q} \geq V$ then

Remove $p$ and $q$ from the clustering procedure;

$\mathrm{m}=\mathrm{m}-1$;

else

In the distance matrix, update the distances between the cluster $(p, q)$ and the other remaining clusters; end

else

Forbid merging $p$ and $q$;

end

else

Forbid merging $p$ and $q$;

end

else

Break;

end 


\subsubsection{Model parameters}

I Set of plant indices $(i=1, \ldots, I)$

$\mathbf{J} \quad$ Set of DC indices $(j=1, \ldots, J)$

Q Set of customer cluster indices $(q=1, \ldots, Q)$

$G=(V, A)$ A graph where $V$ is the set of nodes and $A$ is the set of arcs. $V=V_{P L} \cup V_{D C} \cup V_{C L}$ where $V_{P L}$ is the set of plant nodes, $V_{D C}$ is the set of DC nodes and $V_{C L}$ is the set of cluster nodes. $A=A_{P R} \cup A_{S E}$ where $A_{P R}=\left\{(i, j) \in A \mid i \in V_{P L}, j \in V_{D C}\right\}$ and $A_{S E}=\left\{(j, q) \in A \mid j \in V_{D C}, q \in V_{C L}\right\}$

$D_{q i} \quad$ Total demand of cluster $q$ for the product manufactured in plant $i$ during the whole planning horizon.

totProd $_{i} \quad$ Total volume of cars produced by plant $i$.

$\operatorname{minVol}_{j} \quad$ Minimum volume of cars that has to go through DC $j$ if it is chosen.

$\operatorname{maxVol}_{j} \quad$ Maximum volume of cars that can go through DC $j$ if it is chosen.

$P T C_{i j} \quad$ Cost of a truck going from plant $i$ to DC $j$ (Primary Transport Cost).

$S T C_{j q} \quad$ Cost of a truck starting its route at DC $j$ and visiting all the customers of cluster $q$ before going back to $j$ (Secondary Transport Cost).

$T C_{j} \quad$ Unit transit cost for a car going through DC $j$.

$W_{i} \quad$ Average truck capacity for the cars manufactured in plant $i$.

$W \quad$ Average truck capacity for the whole volume of cars.

$M \quad$ Big value, $M=\min \left(\max \operatorname{lol}_{j}\right.$, totProd $\left._{i}\right)$

NWD Number of working days in the planning horizon.

$T_{\max }(i) \quad$ Maximum waiting time allowed at plant $i$ before shipping to distribution centres.

$T \quad$ Maximum waiting time allowed at a distribution centre before shipping to car dealers.

$C D \quad$ Maximum covering distance parameter (i.e. the maximum length of a route starting at a DC, visiting the customers of a given cluster then coming back to the DC).

$P I_{i j} \quad$ Low volume penalty amount for primary transport from plant $i$ to DC $j$.

$R(j, q) \quad$ Length of the optimal route starting at DC $j$ and visiting the customers of cluster $q$ before coming back to $j$.

$V_{\min }(i) \quad$ Minimum volume of cars that has to go through any opened primary transport link starting at plant $i$. It has to be equal at least to the minimum volume ensuring on average a full truckload within $T_{\max }(i)$ from plant i to a given DC hence $V_{\min }(i) \geq \frac{W_{i}}{T_{\max }(i)} N W D$

$V_{\text {min }} \quad$ Minimum volume of cars that has to go through any opened secondary transport link. It has to be equal at least to the minimum volume ensuring on average a full truckload within $T$ from a DC to any cluster of customers hence $V_{\min } \geq \frac{W}{T} N W D$.

\subsubsection{Decision variables}

- Location variables $y: y_{j}=1$ if $\mathrm{DC} j$ is selected, $y_{j}=0$ otherwise

- Assignment variables $x: x_{i j q}=1$ if cluster $q$ is assigned to DC $j$ for the products of plant $i, x_{i j q}=0$ otherwise 
Minimize:

$$
\begin{array}{rlr}
\text { Total distribution cost } & =\sum_{i \in \mathbf{I}} \sum_{j \in \mathbf{J}} \frac{P T C_{i j}}{W_{i}} \sum_{\substack{q \in \mathbf{Q} \\
R(j, q) \leq C D}} D_{q i} x_{i j q} & \text { (Primary transport cost }=\text { unit cost } \times \text { volume }) \\
& +\sum_{j \in \mathbf{J}} \sum_{\substack{q \in \mathbf{Q} \\
R(j, q) \leq C D}} \frac{S T C_{j q}}{W} \sum_{i \in \mathbf{I}} D_{q i} x_{i j q} & \text { (Secondary transport cost }=\text { unit cost } \times \text { volume }) \\
& +\sum_{j \in \mathbf{J}} T C_{j} \sum_{i \in \mathbf{I}} \sum_{\substack{q \in \mathbf{Q} \\
R(j, q) \leq C D}} D_{q i} x_{i j q} & \text { (Transit cost }=\text { unit cost } \times \text { volume }) \\
& +\sum_{i \in \mathbf{I}} \sum_{j \in \mathbf{J}} P I_{i j} v_{i j}^{\prime \prime} & \text { (Low volume penalties for primary transport })
\end{array}
$$


Subject to:

$$
\begin{aligned}
& \sum_{\substack{j \in \mathbf{J} \\
R(j, q) \leq C D}} x_{i j q}=1 \\
& \sum_{q \in \mathbf{Q}} D_{q i} x_{i j q}=v_{i j}^{\prime}-v_{i j}^{\prime \prime} \\
& v_{i j}^{\prime} \geq V_{\min }(i) z_{i j} \\
& v_{i j}^{\prime} \leq M z_{i j} \\
& v_{i j}^{\prime \prime} \leq V_{\min }(i) z_{i j} \\
& \sum_{i \in \mathbf{I}} \sum_{\substack{q \in \mathbf{Q} \\
R(j, q) \leq C D}} D_{q i} x_{i j q} \geq \operatorname{minVol}_{j} y_{j} \\
& \sum_{i \in \mathbf{I}} \sum_{\substack{q \in \mathbf{Q} \\
R(j, q) \leq C D}} D_{q i} x_{i j q} \leq \max \operatorname{mol}_{j} y_{j} \\
& \sum_{i \in \mathbf{I}} D_{q i} x_{i j q} \geq V_{m i n} a_{j q} \\
& \sum_{i \in \mathbf{I}} x_{i j q} \leq I a_{j q} \\
& z_{i j} \leq y_{j} \\
& y_{j}, x_{i j q}, a_{j q}, z_{i j} \in\{0,1\} \\
& v_{i j}^{\prime}, v_{i j}^{\prime \prime} \geq 0
\end{aligned}
$$$$
\forall i \in \mathbf{I}, q \in \mathbf{Q} ; D_{q i} \geq 0
$$$$
\forall i \in \mathbf{I}, j \in \mathbf{J}
$$$$
\forall i \in \mathbf{I}, j \in \mathbf{J}
$$$$
\forall i \in \mathbf{I}, j \in \mathbf{J}
$$$$
\forall i \in \mathbf{I}, j \in \mathbf{J}
$$$$
\forall j \in \mathbf{J}
$$$$
\forall j \in \mathbf{J}
$$$$
\forall j \in \mathbf{J}, q \in \mathbf{Q} ; R(j, q) \leq C D
$$$$
\forall j \in \mathbf{J}, q \in \mathbf{Q} ; R(j, q) \leq C D
$$$$
\forall i \in \mathbf{I}, j \in \mathbf{J}
$$$$
\forall i \in \mathbf{I}, j \in \mathbf{J}, q \in \mathbf{Q} ; R(j, q) \leq C D
$$$$
\forall i \in \mathbf{I}, j \in \mathbf{J}
$$

The objective function consists in the total distribution cost, i.e. primary transport cost, secondary transport cost, transit cost and penalties for violating the minimum volume constraints on primary transport links. Each of the transport and transit cost components are computed as the unit cost per car multiplied by the volume of cars on each link/DC. Notice that binary variables $y, z$ and $a$ are not used in the objective function as they are created only to express minimum volume constraints for DCs and transport links. Constraints (1) state that the demand of cluster $q$ for each product of plant $i$ is satisfied and is routed through a single DC (as $x$ are binary variables). Constraints (2)-(5) enable us to compute the violation of minimum volume constraints and to penalize it in the objective function. Constraints (6) state that if DC $j$ is selected $\left(y_{j}=1\right)$, then the flows going through $j$ have to be greater than the corresponding minimum volume limit. Constraints (7) stipulate that:

- If DC $j$ is selected $\left(y_{j}=1\right)$ then the flows going through $j$ must not exceed its maximum capacity.

- If DC $j$ is not selected $\left(y_{j}=0\right)$ then there is no flow transiting by it (all $x_{i j q}$ have to be set equal to 0 ).

Constraints (8) ensure that if the link between $j$ and $q$ is selected $\left(a_{j q}=1\right)$ then the corresponding total volume has to be greater than the minimum volume $V_{\text {min }}$. Constraints (9) stipulate that if the link between $j$ and $q$ is not selected $\left(a_{j q}=0\right)$ then all of the variables $x_{i j q}$ have to be set equal to 0 . Constraints (10) stipulate that if DC $j$ is not opened $\left(y_{j}=0\right)$ then all of the variables $z_{i j}$ have to be set equal to 0. Constraints (11) and (12) are the integrality and non negativity constraints. 
Notice that maximum covering distance constraints are implicitly modeled when defining assignment variables $x_{i j q}$. In fact, assignment of cluster $q$ to DC $j$ for the products of plant $i$ is not allowed if the route distance between $j$ and $q$ exceeds the maximum covering distance $C D$.

\section{Heuristic solution approach}

In this section, we propose a heuristic solution approach for the MIP problem presented in the previous modeling section. For small-size instances of the problem, we can use a MIP commercial solver such as CPLEX to obtain an optimal solution. However, computation times become prohibitively high for large-size real-life instances not only for getting optimal solutions but even for getting the first feasible solutions. Therefore, we had to consider the development of a specific and possibly more efficient heuristic solution method.

\subsection{The main idea of the heuristic approach}

The main idea of the proposed heuristic method is to exploit as much as possible the information provided by the optimal solution of the linear relaxation of the problem. An argument supporting this approach is the tightness of the lower bounds provided by the linear relaxation solution of the earlier defined formulation (typical deviations are less than $1.3 \%$ ).

In the problem under study, we have two main types of binary variables: location variables and assignment variables. We propose a two-stage heuristic method: the first stage (S1) focuses on location decisions whereas the second one (S2) deals with assignment decisions. The location variables are fixed in the first stage and the corresponding values are used in the second stage. Three different heuristic methods are implemented for the second stage.

\subsection{First stage (S1): location decisions}

In the first stage, we focus on deciding which DCs should be opened, i.e. we aim at determining the value of each location variable $y$ in such a way as to be as close as possible to the optimal solution. To this end, we propose to solve a partial linear relaxation of the original MIP where the integrality constraints on variables $x, z$ and $a$ have been removed whereas they have been kept for $y$ variables. Different tests showed that this is an efficient way to quickly identify the relevant main structure of the distribution network, i.e. to determine the DCs to open.

\subsection{Second stage (S2): assignment decisions}

In the second stage, we focus on deciding which DCs should serve which clusters, i.e. we aim at determining the value of each assignment variable $x$ in such a way as to meet the minimum volume constraints for secondary transport. After fixing the location variables in the first stage of the heuristic approach, the resulting assignment problem is easier to solve than the original MIP. A rather large fraction (on average 44\%) of the variables $x$ are indeed set to 0 due to the fact that the corresponding DCs are not opened. However, this second-stage problem is not a standard assignment problem: since it includes knapsack constraints (due to capacity restrictions when assigning customers to DCs), it can be shown to be NP-Hard (NP-Hardness of the whole distribution network design problem was already settled in [14]).

In order to solve the second-stage assignment problem, three distinct methods were studied. 


\subsubsection{Reintroduction of all the integrality constraints (S2M1)}

The method S2M1 reintroduces the integrality constraints for all the free assignment variables $x$ just after the location stage then solves the resulting MIP using a commercial solver. This is much quicker than directly solving the original MIP as the number of binary variables is reduced after fixing the location variables.

\subsubsection{Gradual reintroduction of the integrality constraints (S2M2)}

The method S2M2 consists in gradually reintroducing the relaxed integrality constraints. As previously mentioned, in the first stage, we fix the location variables $y$. Then, we add the integrality constraints to all the secondary transport related variables, namely, $x$ and $a$. We solve the resulting MIP using a commercial solver and we fix $x$ and $a$ at their values in the obtained solution. Finally, we add the integrality constraints to all the primary transport related variables (i.e. $z$ ) and we solve the resulting problem. Here, it is worth pointing out that the last step does not change any assignment decision as the $x$ and $a$ variables are fixed in the previous step. It only re-evaluates the objective function taking into account the penalty resulting from violating the minimum volume constraints on primary transport links.

\subsubsection{Fixing strategy (S2M3)}

We now discuss a fixing strategy exploiting the information provided by the solution of the first stage of the heuristic. The idea is to try to fix as many assignment variables as possible before reintroducing the integrality constraints for the currently free variables and solving the resulting MIP with a commercial solver. The fixing strategy should enable us to decrease the number of binary variables before solving the final MIP and thus to reduce computation times in the second stage of the heuristic procedure. However, a key issue is to ensure that the fixing decisions thus made do not lead to infeasibility.

In order to implement the fixing strategy, we started at the optimal solution of the first location stage that we examined to identify the main issues. We noticed two problems: firstly, fractional values are obtained for only a small proportion of the assignment variables (of about $2 \%$ ); secondly, there are of about $50 \%$ of the secondary transport links where the minimum volume constraint is violated. These figures clearly show that the violation of the minimum volume constraints for secondary transport links is the main issue in the optimal solution of the location stage. This is why the fixing strategy detailed in Algorithm 2 aims at setting to 0 any secondary transport flow $(j, q)$ violating the minimum volume constraint, provided that the problem remains feasible. Thus, we make sure that after closing $(j, q)$, the throughput of $\mathrm{DC} j$ will be kept above its minimum volume $\min \mathrm{ol}_{j}$ and that there is another DC $k$ able to deliver the demand of $q$ without exceeding its maximum volume of throughput $\max$ ol ${ }_{k}$.

We repeat this procedure for all the secondary transport links then we solve the resulting linear problem. In case the problem becomes infeasible, we remove the lately added fixing constraints and stop the loop. Otherwise, we iterate the procedure until there are no further flows that can be fixed at 0 or the problem becomes infeasible. The second step of the fixing strategy is to reintroduce the 0-1 constraints for all the free assignment variables and to solve the resulting MIP with a commercial solver.

As detailed in the literature review, we found only four papers dealing with linear relaxation based heuristics for facility location or network problems with minimum volume constraints. The management of feasibility is among the main differences between our fixing algorithm and the solution methods implemented in these papers. In the 
present work, through the tests introduced before setting flows to 0, we try to keep feasibility while fixing assignment variables. In [31], an infeasible solution is corrected after running the fixing algorithm using a feasibility pump. Melo et al. used in [18] a search in the neighborhood of the infeasible solution. In [6] and [16], the authors do not address the feasibility issues at the end of the heuristic procedure.

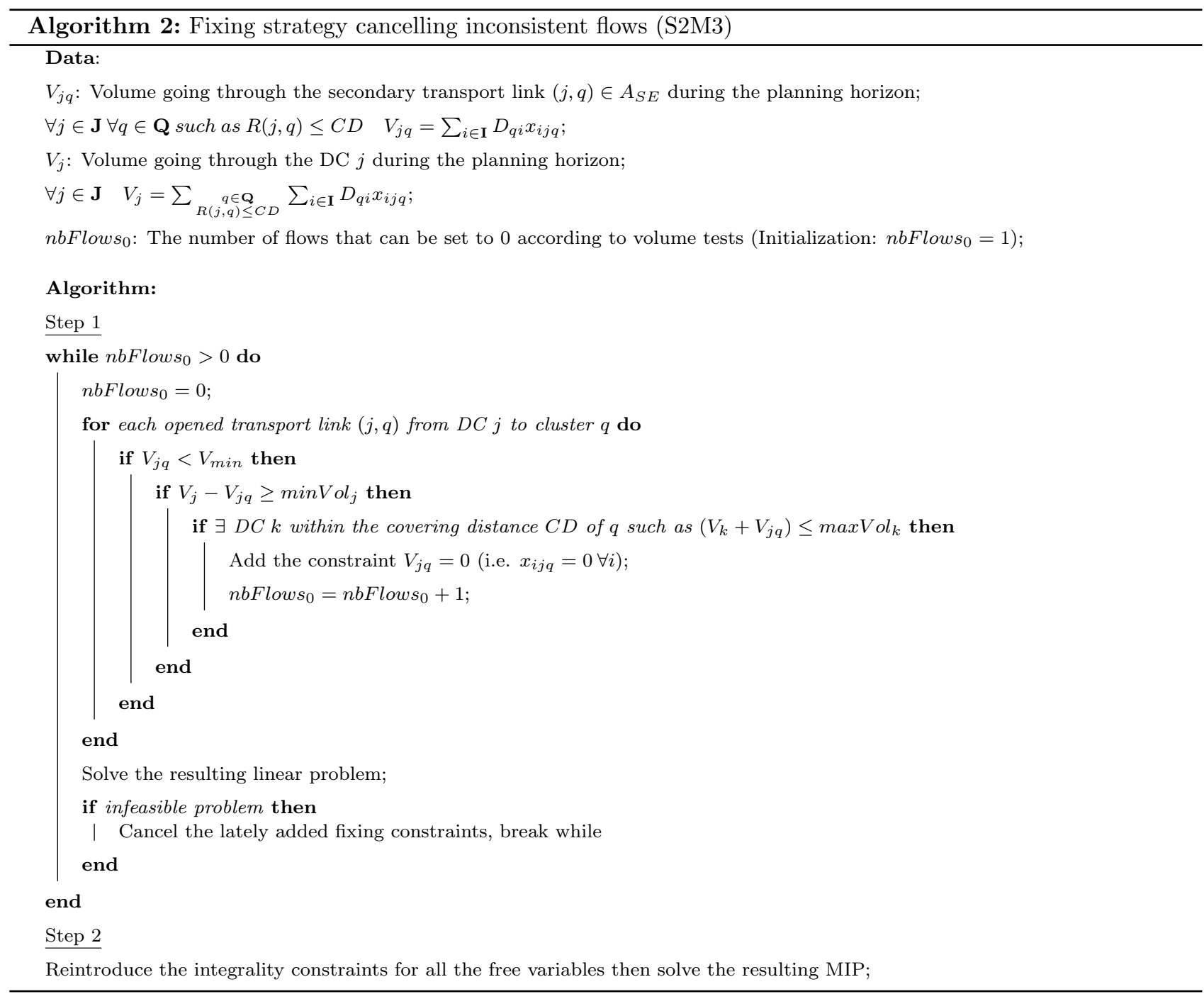

\section{Numerical results}

In this section, we aim at discussing the numerical experiments of our model based on real-life data from our case-study. We employed the $\mathrm{C}++$ language to implement the MIP model and the commercial solver ILOG CPLEX version 12.1 to solve it. We carried out all the tests on a Pentium Core 2 Duo (2.53 GHz) with 1.92Go of RAM, running under Windows XP. After describing the case-study, we analyze the optimal solution given by CPLEX in terms of network configuration (location decisions and different trade-offs) and of computation times. Then, we study the computational performance of the two-stage heuristic approach as compared to applying CPLEX to the original MIP. 


\subsection{Case-study description}

The case-study that motivated this research deals with a car distribution network in France. The network consists of 16 assembly plants (or ports), 51 potential distribution centres and 448 car dealers. We employed a free online tool [7] to obtain the geospatial coordinates of the different sites and the Geographic Information System (GIS) Microsoft MapPoint to evaluate the route distances.

Based on the preferences of the decision maker, we defined a reference dataset where the planning horizon is fixed at one year, the maximum covering distance is set to 460 kilometres. The minimum and maximum volumes per DC are set according to some hypothesis on the flexibility of each DC. The minimum volume per primary transport link is fixed at 1 full truckload within a maximal waiting time of 1 week. The minimum volume per secondary transport link is also fixed at 1 full truckload within a maximal waiting time of 1 week. The clustering parameters are the following: maximum number of customers per cluster $=3$, maximum volume per cluster $=3$ full truckloads within 1 week, maximum distance between any two customers in a cluster $=50$ kilometers and minimum volume per cluster $=2$ full truckloads within 1 week. Here, it is worth pointing out that a difference of 1 truckload between the minimum quantity per cluster and the minimum quantity per secondary transport link gives more flexibility to the optimization algorithm with respect to the possibility of assigning a cluster to several DCs.

\subsection{Test instances}

In order to test the performance of exact and heuristic solutions, we constructed 60 test instances by varying the main parameters of the problem in the reference dataset.

- Instances A.1 to A.12: we vary the value of the maximum covering distance from 460 kilometres to 680 kilometres while fixing the other parameters of the reference dataset. This leads to increasing the number of binary variables (see fig. 4).

- Instances B.1 to B.20: we vary the demand of customers. In each instance, we assign to each customer a different pseudo-random coefficient lying between 0.5 and 1.5. The new customer demand is computed as the reference demand (used in the reference dataset) multiplied by the pseudo-random coefficient.

- Instances C.1 to C.8: we vary the minimum volume parameters for primary transport, secondary transport and DC throughputs as well as the maximum capacities for DCs (see Table. 2). In two instances (C.5 and C.6), we also change the maximum covering distance value because tight minimum volume constraints lead to an infeasible problem.

- Instances D.1 to D.20: each of these instances contains 46 potential DCs instead of 51 as we randomly select 5 DCs to remove from the list of potential DCs.

\subsection{Optimal solution using a commercial solver}

In the present subsection, we study the solution given by the commercial solver CPLEX for the proposed MIP problem. Different tests showed that proving the optimality, in some cases, is very time-consuming. Consequently, we limited the optimality gap of CPLEX to $0.2 \%$ in order to reduce the computational effort (the optimality gap is 


\begin{tabular}{lllllll}
\hline Test instance & $\begin{array}{l}\text { Min volume for pri- } \\
\text { mary transport } \\
\text { truckload/week })\end{array}$ & $\begin{array}{l}\text { Min volume for sec- } \\
\text { ondary transport } \\
\text { truckload/week })\end{array}$ & $\begin{array}{l}\text { Min through- } \\
\text { put per DC }\end{array}$ & $\begin{array}{l}\text { Max through- } \\
\text { put per DC }\end{array}$ & $\begin{array}{l}\text { Max covering distance } \\
(\mathrm{km})\end{array}$ \\
\hline C.1 & 1 & 1 & RefData & RefData & 460 \\
C.2 & 2 & 1 & RefData & RefData & 460 \\
C.3 & 1 & 2 & RefData & RefData & 460 \\
C.4 & 2 & 2 & RefData & RefData & 460 \\
C.5 & 1 & 1 & $1.5 \times$ RefData & RefData & 620 \\
C.6 & 1 & 1 & $2 \times$ RefData & RefData & 660 \\
C.7 & 1 & 1 & RefData & $0.8 \times$ RefData & 460 \\
C.8 & 1 & 1 & RefData & $2 \times$ RefData & 460 \\
\hline
\end{tabular}

Table 2: Test instances C: varying the minimum and maximum volume parameters. RefData denotes the data of the reference dataset.

defined as the relative difference between the solution obtained and the best lower bound found by the Branch \& Bound procedure).

We first explain the setting of the penalty parameter related to primary transport links. Then, we analyze the configuration of the distribution network obtained in the final solution given by CPLEX. Finally, we study the impact of varying the main parameters of the problem on computation times.

\subsubsection{Setting the penalty parameter}

As already suggested in the modeling section, we handle the minimum volume constraints on primary transport links using penalties. We penalize each unit below the targeted minimum volume by a given amount in the objective function. In our context, we can study two alternatives to evaluate this amount. The first one is to set the penalty to a huge value $\left(10^{9}\right.$ for example), which means that the constraint will be violated only when there is no other solution to reach feasibility. The second alternative consists in using a suitable penalty that makes violating the constraint possible only if there is some opportunity to save costs. This is indeed the case if we choose to set the penalty to the unit transport cost. Fig. 3 illustrates the total cost as a function of the transported volume in this case. This situation means that if the volume on a given primary transport link $(i, j) \in A_{P R}$ is greater than the

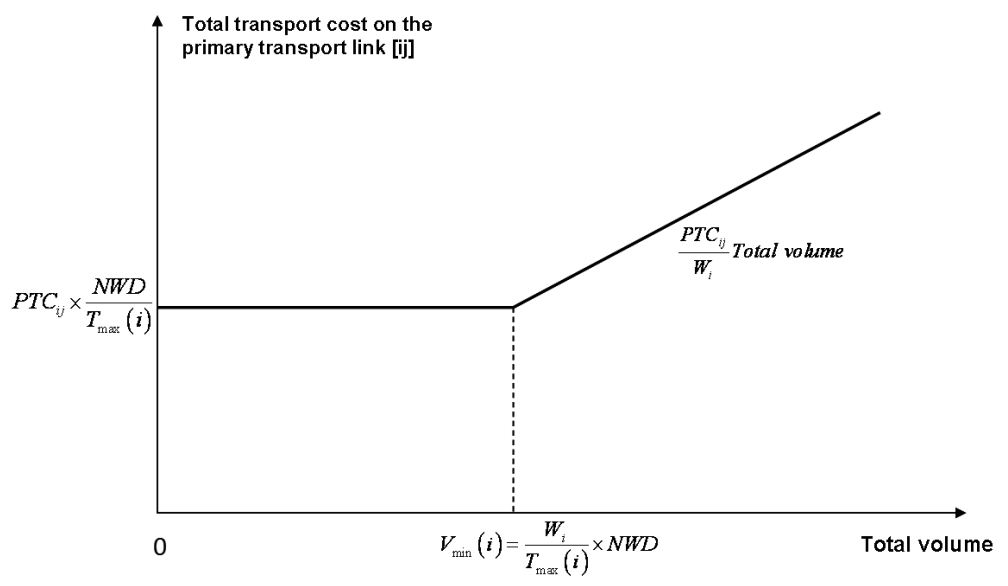

Figure 3: Primary transport cost as a function of the volume 
minimum required volume then all the products can be shipped in full truckloads. We pay a unit transport cost equal to $\frac{P T C_{i j}}{W_{i}}, P T C_{i j}$ is the cost of a truck going from plant $i$ to DC $j$ and $W_{i}$ is the average truck capacity for the trips starting at plant $i$. On the contrary, if the volume on the link is less than the minimum required volume, then regardless to the quantity, we pay a total cost equal to $P T C_{i j} \cdot \frac{N W D}{T_{\max }(i)}$. Observe that this amounts to keep shipping trucks with the same frequency $T_{\max }(i)$ even if they are not fully loaded.

The computational experiments that we carried out using test instances A showed that when the unit penalty is fixed at $10^{9}$, the computation time is prohibitively high regardless of the value of the maximum covering distance (for instance, it is about 14 hours when the maximum distance is set to 460 kilometers and 20 hours when it is equal to 480 kilometers). When the unit penalty is set to the unit transport cost, we found optimal solutions for all the test examples except the one with a maximum distance set to 700 kilometers, that we aborted after 4 days of running time without finding any feasible solution. The computation times remain acceptable until a distance equal to 560 kilometers, after which the running time exceeds 4 hours (see fig. 4)

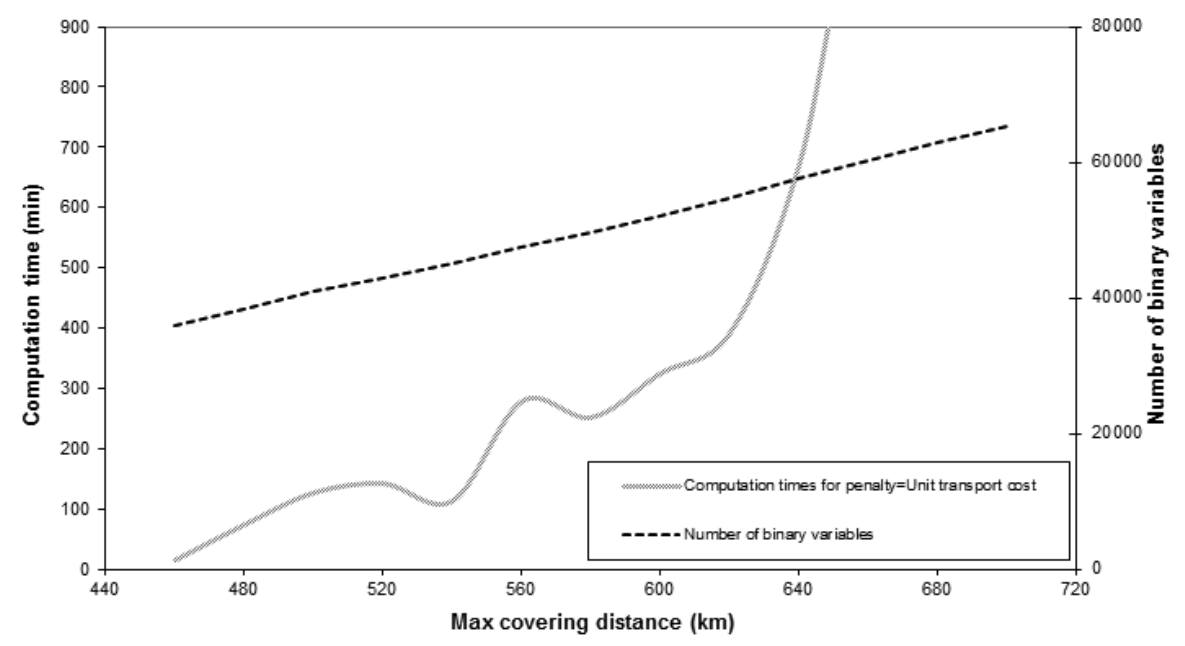

Figure 4: Computation time as a function of the maximum covering distance value for the second penalization alternative

In the subsequent experiments reported in this paper, we fix the penalty value for each primary transport link at the unit transport cost on this link.

\subsubsection{Assessment of the heuristic clustering}

We propose to assess the heuristic clustering presented in $§ 3.2 .2$ by comparing it with an exact clustering based on a set-partitioning formulation (detailed description of this method can be found in [14]). To this aim, we compute on instances B the relative difference in the total distribution cost between a) the optimal solution to the MIP problem (1)-(12) using the heuristic clustering; b) the optimal solution to the MIP problem (1)-(12) using the exact clustering. The values obtained for these instances are displayed in Table. 3. The figures show that the increase in total cost due to the use of heuristic clustering rather than the exact clustering is only about $0.63 \%$ on average, which confirms the good quality of the solutions produced by the heuristic.

We note here that one of the limitations of the exact approach is that the set-partitioning model possibly requires the enumeration of a potentially huge number of candidate clusters (there are more than 60000 of these 
in the instances shown in Table. 3, where no more than 3 customers per cluster are allowed). This may lead to numerically intractable problems when the number of customers or the maximum number of customers per cluster increase. By contrast, an advantage of the heuristic clustering is that it is far less dependent on these two parameters.

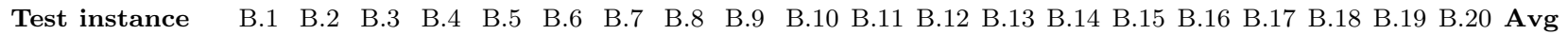

$\begin{array}{llllllllllllllllllllll}\text { Heuristic cost } & 0.82 & 0.64 & 0.50 & 0.42 & 0.62 & 0.52 & 0.53 & 0.85 & 0.49 & 0.83 & 0.64 & 0.19 & 0.66 & 0.44 & 0.66 & 0.66 & 0.92 & 0.98 & 0.46 & 0.81 & 0.63\end{array}$ increase $(\%)$

Table 3: Distribution cost increase when using a heuristic clustering rather than an exact one on instances (B) varying customer demand.

\subsubsection{Distribution network}

The first step of our location-routing procedure is the application of the heuristic clustering algorithm over the 448 customers of the country. This results in 302 clusters meeting the various grouping constraints. Then, we run the network optimization on the reference dataset in order to obtain the best locations for DCs and the assignment of clusters to them. The first conclusion that can be made from the obtained figures is that the number of opened DCs is relatively high: 28 among 51 potential sites. This means that secondary transport considerably influences the network configuration, first because of the maximum covering distance constraint and second as the cost per car per kilometer for secondary transport is higher than the cost for primary transport, due to the difference of truck speed (secondary transport usually concerns last-mile deliveries in urban areas where vehicles are slower). As customers are scattered all over the country, we try to get close to them by opening many DCs. Fig. 5 shows that DCs are specialized by region and that transport flows assigned to them are related to close customers.

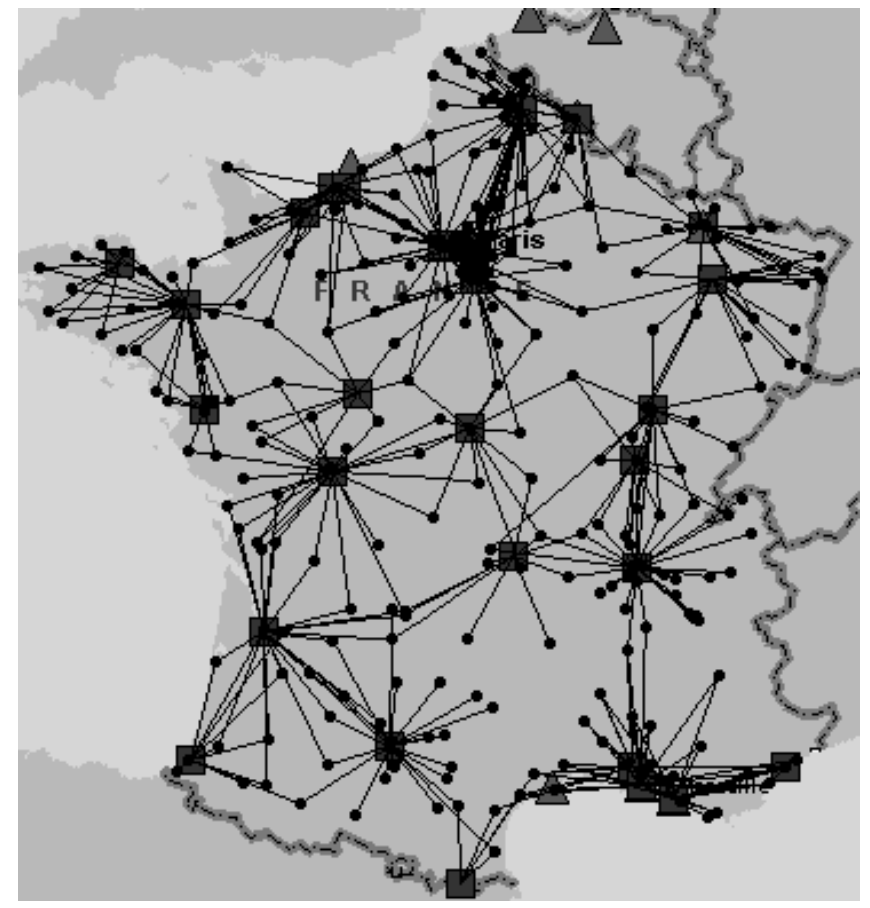

Figure 5: Secondary transport in the optimal solution given by CPLEX. Plant locations are represented using triangles, opened DCs using squares, and cluster barycentres using dark circles. As the figure is focused on secondary transport, some plants do not appear. 
However, DCs were not opened at each potential location as this may violate minimum throughput constraints for DCs. The throughput of many opened DCs is indeed close to the minimum required quantity. This reports on the impact of minimum throughput constraints on the network configuration. Furthermore, the number of DCs assigned to each cluster varies between 1 and 5. Obviously, clusters are more likely to be served by their closest DCs. This is why the number of single assignments is important (34\% of clusters are assigned to only one DC). Other clusters are assigned to several DCs either because the problem is highly constrained or because sometimes it is better to use a farther facility which costs less in terms of primary transport.

\subsubsection{Impact of varying parameters on computation times}

Different tests showed that the following parameters could have a considerable impact on computation times:

- Clustering parameters: Depending on these parameters, the number of clusters resulting from the algorithm could vary and then influences the problem size. The most influential parameter is the minimum volume per cluster $V$. Decreasing $V$ leads to an increased number of clusters and consequently to an increased number of assignment variables $x$.

- Minimum volume parameters: Increasing the minimum volume required for transport links or for the throughput of DCs makes the problem more difficult to solve as it is not easy to find a trade-off between all the constraints. For instance, computation time went from 15 minutes to 35 hours when we doubled the minimum volume required per secondary transport link (2 full truckloads instead of 1 ).

- Maximum covering distance parameter: Increasing the value of the maximum distance parameter leads to increasing the number of possible assignments, which results in raising the number of the assignment variables and of the related constraints. For instance, the size of the problem goes from 35853 binary variables and 13946 constraints when the maximum covering distance is fixed at 460 kilometers to 62832 binary variables and 17120 constraints when the maximum covering distance is set to 680 kilometers. Fig. 4 shows how the computation time significantly increases when the distance increases. If the distance is set to 700 kilometers then the computation time to find a feasible solution can become prohibitive (more than 24 hours in some cases).

Thus, even if the computation time needed by CPLEX MIP solver to solve the problem on the reference dataset is acceptable (15 minutes), the analysis carried out in this paragraph showed that the variation of the main parameters of the problem, in particular the maximum covering distance, can result in extensive running times as the number of integer variables and constraints significantly increases. Moreover, this study has an interactive character: the decision maker wants to run many what-if scenarios and requires to quickly view the impact of certain decisions. This is why implementing a heuristic method appeared to be the best way to find a near-optimal solution in a reasonable runtime, so that the decision making process is facilitated without deteriorating the solution quality.

\subsection{Experiments with the heuristic approach of section 4}

In the present subsection, we study the performance of the heuristic approach investigated in section 4 as compared to the reference solution given by CPLEX for the original MIP. The optimality gap was limited to $0.2 \%$ 
for the reference solution and for all the mixed integer programs solved within the heuristic methods (using CPLEX also). The parameter setting was based on the reference dataset defined in $§ 5.1$.

As previous tests with CPLEX (based on default settings) show some difficulties in finding feasible solutions, we tried to emphasize feasibility over optimality in the branching procedure using the "MIPEMPHASIS" parameter of CPLEX. Nonetheless, this approach did not lead to significant improvements in computation times. We then examined another alternative using the "setPriorities" function, which drives CPLEX to give priority to location variables over assignment variables during branching. Location decisions have indeed a structural role in our network design problem. This change in the settings of CPLEX resulted in a noticeable decrease in the computational effort. The first part of Table. 4 shows computation times in minutes for CPLEX applied to the original MIP both with default settings ( $6^{\text {th }}$ column) and with priorities assigned to location variables ( $7^{\text {th }}$ column) on instances A varying the value of the maximum covering distance.

It is seen that CPLEX behaves much better when using priorities, on average CPU is half the initial value. This is why in the subsequent tests, we will keep using priorities. The resulting figures are compared with those given by heuristic methods (S2M1, S2M2 and S2M3). The second part of Table. 4 shows the solution quality of the three heuristic methods and of the solver output for the original MIP within 10 minutes and 60 minutes respectively. In fact, this information is useful to analyze the performance of the two-stage heuristic approach as compared to the exact one.

Overall, Table. 4 shows that the gradual reintroduction of the integrality constraints in the second stage (S2M2) leads to running times shorter than S2M1 and S2M3 but to higher deviations from the original MIP solutions (up to $1.71 \%$ ). In fact, in the second step of S2M2, we ignore the minimum volume constraints for primary transport links as the corresponding variables could have fractional values. Consequently, some expensive decisions are made for the transport from plants to distribution centres. On the other hand, the computational results clearly show that S2M1 yields excellent quality solutions and sometimes outperforms CPLEX applied to the original MIP (negative values in the second part of Table. 4). This could be explained by the fact that the MIP optimality gap was limited to $0.2 \%$. In return, the computation times obtained with S2M1 could increase up to 133 minutes. Thus, to achieve a trade-off between time and value, we should apply the fixing strategy S2M3, which is indeed better than S2M1 in terms of running time and which solution is less expensive than the one provided by S2M2. According to the choice of the decision maker, it is possible to prioritize the solution speed (S2M2), the solution quality (S2M1) or a trade-off between these two features (S2M3).

In summary, using the two-stage heuristic approach appears to be significantly more competitive than running the solver on the whole MIP model of section 3.4.3. We can indeed notice according to the last two columns of Table. 4 that within 10 minutes CPU, CPLEX cannot find any feasible integer solution. Within 60 minutes CPU, it yields a solution better than the heuristic one only in the first two instances. This conclusion was also validated through additional extensive numerical tests using test instances B, C and D defined in $§ 5.2$. The computational results are summarized in Tables. 5, 6 and 7; the obtained figures again show the good performance of the 2-stage heuristic procedures. 


\begin{tabular}{|c|c|c|c|c|c|c|}
\hline & & \multirow{2}{*}{\multicolumn{3}{|c|}{ Heuristic solution }} & \multirow{2}{*}{\multicolumn{2}{|c|}{ CPLEX applied to the original MIP }} \\
\hline & $\mathrm{CPU}(\min )$ & & & & & \\
\hline Instance & $\begin{array}{l}\text { Max covering distance } \\
(\mathrm{km})\end{array}$ & S2M1 & S2M2 & S2M3 & $\begin{array}{l}\text { Optimality gap set to } 0.2 \% \text {, } \\
\text { default settings }\end{array}$ & $\begin{array}{l}\text { Optimality gap set to } 0.2 \% \text {, } \\
\text { higher priorities for location } \\
\text { variables }\end{array}$ \\
\hline A. 1 & 460 & 0.8 & 0.3 & 0.5 & 15.3 & 8.4 \\
\hline A. 2 & 480 & 0.6 & 0.3 & 0.7 & 74.4 & 22.8 \\
\hline A. 3 & 500 & 2.1 & 0.4 & 0.7 & 127.3 & 28.8 \\
\hline A. 4 & 520 & 1.9 & 0.4 & 0.4 & 142.3 & 76.4 \\
\hline A. 5 & 540 & 5.0 & 0.3 & 0.4 & 113.8 & 41.7 \\
\hline A. 6 & 560 & 6.2 & 0.5 & 0.5 & 279.1 & 255.2 \\
\hline A. 7 & 580 & 6.6 & 0.6 & 0.7 & 252.4 & 202.1 \\
\hline A. 8 & 600 & 19.8 & 0.4 & 0.9 & 325.5 & 209.4 \\
\hline A. 9 & 620 & 20.2 & 0.4 & 0.5 & 392.7 & 159.2 \\
\hline A.10 & 640 & 14.7 & 0.5 & 0.6 & $>10 \mathrm{~h}$ & $>10 \mathrm{~h}$ \\
\hline A.11 & 660 & 15.9 & 0.5 & 0.9 & $>10 \mathrm{~h}$ & $>10 \mathrm{~h}$ \\
\hline \multirow[t]{2}{*}{ A.12 } & 680 & 91.2 & 0.6 & 2.3 & $>10 \mathrm{~h}$ & $>10 \mathrm{~h}$ \\
\hline & Solution quality(\%) & \multicolumn{3}{|c|}{ Heuristic solution } & \multicolumn{2}{|c|}{ CPLEX applied to the original MIP } \\
\hline Instance & $\begin{array}{l}\text { Max covering distance } \\
(\mathrm{km})\end{array}$ & S2M1 & $\mathrm{S} 2 \mathrm{M} 2$ & S2M3 & Time limit set to $10 \mathrm{~min}$ & Time limit set to $60 \mathrm{~min}$ \\
\hline A.1 & 460 & -0.06 & 1.49 & 0.73 & NFS & -0.12 \\
\hline A. 2 & 480 & 0.07 & 1.61 & 0.75 & NFS & 0.03 \\
\hline A. 3 & 500 & 0.06 & 1.54 & 0.70 & NFS & NFS \\
\hline A. 4 & 520 & 0.06 & 1.71 & 0.90 & NFS & NFS \\
\hline A. 5 & 540 & 0.05 & 1.64 & 0.97 & NFS & NFS \\
\hline A. 6 & 560 & -0.01 & 1.70 & 0.97 & NFS & NFS \\
\hline A. 7 & 580 & 0.08 & 1.69 & 0.99 & NFS & NFS \\
\hline A. 8 & 600 & -0.04 & 1.40 & 0.86 & NFS & NFS \\
\hline A. 9 & 620 & -0.06 & 1.36 & 0.87 & NFS & NFS \\
\hline A.10 & 640 & 0.02 & 1.40 & 0.88 & NFS & NFS \\
\hline A.11 & 660 & 0.01 & 1.44 & 0.89 & NFS & NFS \\
\hline A.12 & 680 & 0.03 & 1.42 & 0.92 & NFS & NFS \\
\hline
\end{tabular}

Table 4: CPU (min) and solution quality (\%) as a function of the maximum covering distance (instances A). CPU of CPLEX solutions is mentioned in 2 cases: when using default settings and when higher priorities are assigned to location variables during the branching procedure. Solution quality is measured as the relative difference between the heuristic solution and the solution produced by CPLEX applied to the original MIP within $0.2 \%$ of optimality. The heuristic uses one of the methods $\mathrm{S} 2 \mathrm{M} i, i=1 . .3$ in the $2^{\text {nd }}$ stage. Optimality gap for any MIP used in the heuristics was limited to $0.2 \%$. NFS means that no feasible solution was found within the allowed time limit.

\section{Conclusion and future research}

In this paper, we studied a multi-product distribution network design problem arising in the context of car mulation of a large-size MIP with minimum volume, maximum covering distance and single sourcing constraints. We analyzed the main trade-offs related to the network structure and provided numerical results using real-life data. These results showed that it is possible to handle problems featuring about 500 customers, 15 products, 50 


\begin{tabular}{|c|c|c|c|c|}
\hline \multirow{2}{*}{$\begin{array}{l}\mathrm{CPU}(\min ) \\
\text { Test instance }\end{array}$} & \multicolumn{3}{|c|}{ Heuristic solution } & \multirow{2}{*}{$\begin{array}{l}\text { CPLEX applied to the original MIP } \\
\text { Optimality gap set to } 0.2 \%\end{array}$} \\
\hline & S2M1 & $\mathrm{S} 2 \mathrm{M} 2$ & S2M3 & \\
\hline B.1 & 1.6 & 0.2 & 0.4 & 29.9 \\
\hline B. 2 & 1.0 & 0.3 & 0.2 & 13.0 \\
\hline B.3 & 2.4 & 0.4 & 0.2 & 243.1 \\
\hline B. 4 & 0.9 & 0.3 & 0.2 & 41.6 \\
\hline B. 5 & 0.4 & 0.2 & 0.2 & 8.3 \\
\hline B. 6 & 0.3 & 0.2 & 0.2 & 11.8 \\
\hline B.7 & 1.0 & 0.2 & 0.2 & 11.7 \\
\hline B. 8 & 0.9 & 0.2 & 0.2 & 17.7 \\
\hline B.9 & 1.1 & 0.2 & 0.2 & 22.1 \\
\hline B.10 & 2.3 & 0.5 & 0.3 & 31.5 \\
\hline B.11 & 0.8 & 0.4 & 0.2 & 31.6 \\
\hline B.12 & 1.2 & 0.8 & 0.2 & 19.8 \\
\hline B.13 & 0.3 & 0.2 & 0.2 & 23.3 \\
\hline B.14 & 0.4 & 0.3 & 0.3 & 40.7 \\
\hline B. 15 & 0.9 & 0.5 & 0.3 & 25.0 \\
\hline B. 16 & 2.0 & 0.2 & 0.2 & 67.2 \\
\hline B. 17 & 1.1 & 0.2 & 0.3 & 35.0 \\
\hline B. 18 & 2.2 & 0.2 & 0.2 & 19.2 \\
\hline B.19 & 0.9 & 0.2 & 0.2 & 20.3 \\
\hline B. 20 & 0.7 & 0.2 & 0.3 & 16.3 \\
\hline Avg & 1.1 & 0.3 & 0.2 & 36.5 \\
\hline Solution quality(\%) & \multicolumn{4}{|c|}{ Heuristic solution } \\
\hline Test instance & $\mathrm{S} 2 \mathrm{M} 1$ & $\mathrm{~S} 2 \mathrm{M} 2$ & S2M3 & \\
\hline B.1 & 0.10 & 1.03 & 0.61 & \\
\hline B. 2 & -0.03 & 1.51 & 0.82 & \\
\hline B.3 & 0.09 & 1.75 & 0.63 & \\
\hline B. 4 & -0.03 & 1.19 & 0.72 & \\
\hline B.5 & 0.06 & 1.50 & 0.93 & \\
\hline B. 6 & -0.07 & 1.25 & 0.63 & \\
\hline B.7 & 0.07 & 1.40 & 0.70 & \\
\hline B. 8 & 0.01 & 1.93 & 0.70 & \\
\hline B.9 & -0.08 & 1.37 & 0.49 & \\
\hline B.10 & -0.07 & 1.55 & 0.69 & \\
\hline B.11 & 0.08 & 1.40 & 0.51 & \\
\hline B.12 & 0.04 & 1.57 & 0.86 & \\
\hline B.13 & 0.08 & 1.41 & 0.74 & \\
\hline B.14 & 0.04 & 1.43 & 0.72 & \\
\hline B. 15 & 0.01 & 1.51 & 0.34 & \\
\hline B. 16 & 0.06 & 1.37 & 0.64 & \\
\hline B.17 & 0.02 & 1.43 & 0.83 & \\
\hline B. 18 & 0.06 & 1.68 & 0.54 & \\
\hline B.19 & -0.03 & 1.66 & 0.53 & \\
\hline B. 20 & 0.02 & 1.46 & 0.64 & \\
\hline Avg & 0.02 & 1.47 & 0.66 & \\
\hline
\end{tabular}

Table 5: CPU (min) and solution quality (\%) when varying customer demand (test instances B). 


\begin{tabular}{lllll}
\hline CPU(min) & \multicolumn{2}{l}{ Heuristic solution } & \multicolumn{2}{l}{ CPLEX applied to the original MIP } \\
\hline Test instance & S2M1 & S2M2 & S2M & Optimality gap set to 0.2\% \\
\hline C.1 & 0.8 & 0.3 & 0.5 & 8.4 \\
C.2 & 7.4 & 0.2 & 1.0 & 56.4 \\
C.3 & 8.7 & 0.6 & 1.0 & 551.9 \\
C.4 & 1431.9 & 0.9 & 8.8 & OOM \\
C.5 & 6.6 & 3.0 & 1.2 & 377.7 \\
C.6 & OOM & 3.9 & 8.7 & OOM \\
C.7 & 1.7 & 0.2 & 0.2 & 19.3 \\
C.8 & 1.2 & 0.1 & 0.2 & 25.9 \\
\hline Solution quality(\%) & Heuristic solution & & \\
\hline Test instance & S2M1 & S2M2 & S2M3 & \\
\hline C.1 & -0.06 & 1.49 & 0.73 & \\
C.2 & 0.06 & 6.06 & 1.88 & \\
C.3 & 0.01 & 1.17 & 1.24 & \\
C.4 & - & - & - & \\
C.5 & 0.04 & 1.00 & 0.43 & \\
C.6 & - & - & - & \\
C.7 & -0.03 & 1.27 & 0.67 & \\
C.8 & 0.01 & 1.25 & 0.89 & \\
Avg & $\mathbf{0 . 0 1}$ & $\mathbf{2 . 0 4}$ & $\mathbf{0 . 9 7}$ & \\
\hline
\end{tabular}

Table 6: CPU (min) and solution quality (\%) when varying the minimum and maximum volume parameters (test instances C). OOM denotes an out of memory status without finding any feasible solution and "-" means that the solution quality cannot be evaluated as no reference solution was found. 


\begin{tabular}{|c|c|c|c|c|}
\hline \multirow{2}{*}{$\begin{array}{l}\mathrm{CPU}(\min ) \\
\text { Test instance }\end{array}$} & \multicolumn{3}{|c|}{ Heuristic solution } & \multirow{2}{*}{$\begin{array}{l}\text { CPLEX applied to the original MIP } \\
\text { Optimality gap set to } 0.2 \%\end{array}$} \\
\hline & S2M1 & $\mathrm{S} 2 \mathrm{M} 2$ & S2M3 & \\
\hline D.1 & 1.1 & 0.7 & 0.7 & 26.4 \\
\hline D. 2 & 2.4 & 0.2 & 0.2 & 103.6 \\
\hline D. 3 & 0.4 & 0.3 & 0.3 & 32.7 \\
\hline D.4 & 10.9 & 0.2 & 0.5 & 47.2 \\
\hline D. 5 & 0.2 & 0.2 & 0.2 & 7.3 \\
\hline D. 6 & 1.1 & 0.2 & 0.2 & 6.8 \\
\hline D.7 & 0.2 & 0.2 & 0.2 & 6.2 \\
\hline D. 8 & 0.6 & 0.2 & 0.2 & 5.7 \\
\hline D.9 & 0.5 & 0.3 & 0.2 & 8.0 \\
\hline D.10 & 1.0 & 0.2 & 0.2 & 5.4 \\
\hline D.11 & 0.6 & 0.2 & 0.1 & 5.7 \\
\hline D.12 & 0.5 & 0.3 & 0.3 & 11.4 \\
\hline D.13 & 0.6 & 0.2 & 0.2 & 1.6 \\
\hline D.14 & 0.8 & 0.2 & 0.5 & 12.4 \\
\hline D. 15 & 0.9 & 0.2 & 0.2 & 4.9 \\
\hline D.16 & 0.2 & 0.2 & 0.2 & 2.2 \\
\hline D.17 & 0.4 & 0.1 & 0.1 & 6.5 \\
\hline D.18 & 0.9 & 0.2 & 0.2 & 22.3 \\
\hline D.19 & 0.9 & 0.4 & 0.2 & 3.6 \\
\hline D. 20 & 0.6 & 0.2 & 0.2 & 23.6 \\
\hline Avg & 1.2 & 0.2 & 0.2 & 17.2 \\
\hline Solution quality $(\%)$ & \multicolumn{4}{|c|}{ Heuristic solution } \\
\hline Test instance & $\mathrm{S} 2 \mathrm{M} 1$ & $\mathrm{~S} 2 \mathrm{M} 2$ & $\mathrm{~S} 2 \mathrm{M} 3$ & \\
\hline D.1 & 0.08 & 1.56 & 0.56 & \\
\hline D. 2 & -0.01 & 1.20 & 0.70 & \\
\hline D. 3 & 0.09 & 0.87 & 0.47 & \\
\hline D.4 & 0.08 & 1.65 & 0.57 & \\
\hline D.5 & -0.001 & 0.89 & 0.44 & \\
\hline D. 6 & 0.09 & 1.32 & 0.75 & \\
\hline D.7 & 0.002 & 0.85 & 0.61 & \\
\hline D. 8 & -0.05 & 1.01 & 0.64 & \\
\hline D.9 & 0.04 & 1.15 & 0.81 & \\
\hline D.10 & 0.06 & 1.29 & 0.82 & \\
\hline D.11 & -0.05 & 0.99 & 0.69 & \\
\hline D.12 & 0.02 & 0.69 & 0.42 & \\
\hline D.13 & 0.04 & 0.88 & 0.75 & \\
\hline D. 14 & -0.07 & 1.47 & 0.71 & \\
\hline D. 15 & -0.02 & 1.16 & 0.41 & \\
\hline D.16 & -0.04 & 1.02 & 0.65 & \\
\hline D. 17 & -0.04 & 0.69 & 0.62 & \\
\hline D.18 & 0.003 & 1.14 & 0.74 & \\
\hline D.19 & -0.07 & 1.10 & 0.68 & \\
\hline D. 20 & 0.05 & 1.12 & 0.78 & \\
\hline Avg & 0.01 & 1.10 & 0.64 & \\
\hline
\end{tabular}

Table 7: CPU(min) and solution quality(\%) when varying the list of potential DCs (test instances D) 
potential locations for DCs and, for each product, an average of 6 possible assignments of customers to DCs. The corresponding MIP models, which can be solved within $0.2 \%$ accuracy in less than 2 hours CPU, typically involve 45100 0-1 variables and 15000 constraints.

In order to improve the computational performance of the solution procedure, several heuristic methods using various relaxations of the original MIP formulation of the problem have been described. They were validated through extensive computational experiments where the produced solutions have been compared with those obtained using an efficient state-of-the-art exact MIP solver. To the best of our knowledge, it is the first time that this kind of approach is applied to determine both optimal location and assignment decisions in a supply chain network design problem subject to minimum volume and distance constraints. All the related papers that we found in the literature worked only on rounding the location decisions. The results of our computational experiments confirm the good performance of the proposed heuristic approaches even for the hardest case (62832 0-1 variables and 17120 constraints). Good quality feasible solutions are consistently obtained within short computation times on instances for which a state-of-the-art MIP solver does not produce any feasible solution.

In terms of future research, it would be interesting to study other possible approaches to constructing heuristic solutions. For instance, within the fixing strategy, one such possibility would be to accept infeasible solutions and to repair them instead of building only feasible solutions. Another challenging option that is worth being considered for future research is to look at a dynamic or multi-scenario version of the current model in order to deal with demand seasonality. Various scenarios of demand could indeed have different impact on the network structure and in particular on the major decision variables related to distribution centre location. Moreover, this extension of the model is likely to take full benefit from the good performance of our two-stage heuristic approach especially designed for tackling problems of larger sizes.

\section{References}

[1] M. Albareda-Sambola, E. Fernandez, and G. Laporte. The capacity and distance constrained plant location problem. Computers and Operations Research 36 pp. 597-611, 2009.

[2] S. A. Alumur, S. Nickel, F. Saldanha da Gama, and V. Verter. Multi-period reverse logistics network design. European Journal of Operational Research 220 pp. 67-78, 2012.

[3] N. Azad and H. Davoudpour. A hybrid tabu-SA algorithm for location-inventory model with considering capacity levels and uncertain demands. Journal of Information and Computing Science 3 No. 4 pp. 290-304, 2008.

[4] R. Baldacci, A. Mingozzi, and R. Wolfler Calvo. An exact method for the capacitated location-routing problem. Operations Research 59 N5 pp. 1284-1296, 2011.

[5] S. Barreto, C. Ferreira, J. Paixão, and B. Sousa Santos. Using clustering analysis in a capacitated locationrouting problem. European Journal of Operational Research 179 pp. 968-977, 2007.

[6] A. Barros, R. Dekker, and V. Scholten. A two-level network for recycling sand: a case study. European Journal of Operational Research 110 pp. 199-214, 1998. 
[7] BatchGeocodeur. http://www.batchgeocodeur.mapjmz.com/, accessed 05/20/2014.

[8] I. Correia, T. Melo, and F. Saldanha da Gama. Comparing classical performance measures for a multi-period, two-echelon supply chain network design problem with sizing decisions. Computers and Industrial Engineering 64 pp. 366-380, 2013.

[9] CSCMP. 23rd annual "state of logistics" report. Council of Supply Chain Management Professionals, 2012.

[10] R. Z. Farahani, N. Asgari, N. Heidari, M. Hosseininia, and M. Goh. Covering problems in facility location: a review. Computers and Industrial Engineering 62 pp. 368-407, 2012.

[11] A. Geoffrion and G. Graves. Multicommodity distribution system design by Benders decomposition. Management Science 20 No.5 pp. 822-844, 1974.

[12] S. Guha, A. Meyerson, and K. Munagala. Hierarchical placement and network design problems. FOCSOO Proceedings of the 41st annual symposium on foundations of computer science pp. 603, 2000.

[13] D. R. Karger and M. Minkoff. Building Steiner trees with incomplete global knowledge. FOCS00 Proceedings of the 41st annual symposium on foundations of computer science pp. 613, 2000.

[14] M. Kchaou Boujelben. Modeling and solving a distribution network design problem with multiple operational constraints. Application to a case-study in the automotive industry. Ph.D. Dissertation, Department of Industrial Engineering, Ecole Centrale Paris, 2013.

[15] S. O. Krumke and C. Thielen. Minimum cost flows with minimum quantities. Information processing letters 111 pp. 533-537, 2011.

[16] A. Lim, W. Fan, and Z. Xu. A transportation problem with minimum quantity commitments. Transportation science 40 No. 1 pp. 117-129, 2006.

[17] M. T. Melo, S. Nickel, and F. Saldanha da Gama. Dynamic multi-commodity capacitated facility location: a mathematical modeling framework for strategic supply chain planning. Computers and Operations Research 33 pp. 181-208, 2005.

[18] M. T. Melo, S. Nickel, and F. Saldanha da Gama. An efficient heuristic approach for a multi-period logistics network redesign problem. TOP pp. 1-29, 2011.

[19] A. Meyerson. Profit-earning facility location. STOC01 pp. 30-36, Crete Greece, Heraklion, July 6-8, 2001.

[20] I. D. Moon and S. S. Chaudhry. An analysis of network location problems with distance constraints. Management Science 30 N3 pp. 290-307, 1984.

[21] I. D. Moon and L. Papayanopoulos. Facility location on a tree with maximum distance constraints. Computers and Operations Research 22 N9 pp. 905-914, 1995.

[22] G. Nagy and S. Salhi. Location-routing: Issues, models and methods. European Journal of Operational Research 117, pp. 649-672, 2008. 
[23] M. Ndiaye and H. Alfares. Modeling healthcare facility location for moving population groups. Computers and Operations Research 35 pp. 2154-2161, 2008.

[24] C. Prodhon and C. Prins. A survey of recent research on location-routing problems. European Journal of Operational Research In Press, 2014.

[25] C. ReVelle, H. Eiselt, and M. Daskin. A bibliography for some fundamental problem categories in discrete location science. European Journal of Operational Research 184 pp.817-848, 2008.

[26] E. H. Sabri and B. M. Beamon. A multi-objective approach to simultaneous strategic and operational planning in supply chain design. Omega 28 pp. 581-598, 2000.

[27] J. Sáez-Aguado and P. C. Trandafir. Some heuristic methods for solving p-median problems with a coverage constraint. European Journal of Operational Research 220 pp. 320-327, 2012.

[28] H. G. Seedig. Network flow optimization with minimum quantities. In B.Hu, K. Morasch, S. Pickl, and M. Siegle, editors, Operations Research Proceedings 2010, Operations Research Proceedings, pp. 295-300. Springer Berlin Heidelberg, 2011.

[29] Z. Shen and L. Qi. Incorporating inventory and routing costs in strategic location models. European Journal of Operational Research 179 pp.372-389, 2007.

[30] Z. Svitkina. Lower-bounded facility location. ACM Transactions on algorithms (TALG) Volume 6 Issue 4, 2010.

[31] P. N. Thanh, O. Péton, and N. Bostel. A linear relaxation-based heuristic approach for logistics network design. Computers and Industrial engineering 59 pp. 964-975, 2010.

[32] C. J. Vidal and M. Goetschalckx. Strategic production distribution models: A critical review with emphasis on global supply chain models. European Journal of Operational Research 98 pp. 1-18, 1997.

[33] T.-H. Wu, C. Low, and J.-W. Bai. Heuristic solutions to multi-depot location-routing problems. Computers and Operations Research 29 pp. 1393-1415, 2002.

[34] X. Zhu, Q. Yuan, A. Garcia-Diaz, and L. Dong. Minimal-cost network flow problems with variable lower bounds on arc flows. Computers and Operations Research 38 pp. 1210-1218, 2011. 